\title{
Downregulation of $d r s$ tumor suppressor gene in highly malignant human pulmonary neuroendocrine tumors
}

\author{
MISUZU SHIMAKAGE ${ }^{1}$, KEN KODAMA ${ }^{2}$, KUNIMITSU KAWAHARA ${ }^{3}$, CHUL JANG KIM $^{4}$, \\ YOSHIHIKO IKEDA ${ }^{5}$, MASUO YUTSUDO ${ }^{6}$ and HIROKAZU INOUE ${ }^{7}$
}

\author{
${ }^{1}$ Department of Pediatrics, National Hospital Organization, Wakayama National Hospital, Mihama-cho, \\ Hidaka-gun, Wakayama 644-0044; ${ }^{2}$ Department of Thoracic Surgery, Osaka Medical Center for Cancer and \\ Cardiovascular Diseases, Nakamichi, Higashinari-ku, Osaka 537-8511; ${ }^{3}$ Department of Pathology, Osaka Prefectural \\ Medical Center for Respiratory and Allergic Diseases, Habikino, Osaka 583-8588; ${ }^{4}$ Department of Urology, \\ Kohka Public Hospital, Kohka, Shiga 528-0014; ${ }^{5}$ Department of Pathology, National Cardiovascular Center, \\ Fujishirodai, Suita, Osaka 565-8565; ${ }^{6}$ Division of Cancer and Developmental Biology, Research Institute \\ for Microbial Diseases, Osaka University, Yamadaoka, Suita, Osaka 565-0871; ${ }^{7}$ Department of Microbiology, \\ School of Medicine, Shiga University of Medical Science, Setatsukinowa-cho, Ohtsu, Shiga 520-2192, Japan
}

Received September 30, 2008; Accepted December 29, 2008

DOI: 10.3892/or_00000362

\begin{abstract}
Neuroendocrine tumors in the lung fall into four categories: typical carcinoid tumor (TC), atypical carcinoid tumor (AC), large-cell neuroendocrine carcinoma (LCNEC) and small-cell lung carcinoma (SCLC), in ascending order of malignancy. The $d r s$ gene was originally isolated as a suppressor against v-src transformation and was shown to induce apoptosis in human cancer cells. The expression of $d r s$ was markedly downregulated in various human cancer tissues and cell lines. Furthermore, drs knockout mice showed a tumor-prone phenotype, indicating that $d r s$ acts as a tumor suppressor gene in malignant tumor formation. To clarify the role of the $d r s$ gene in the development of human pulmonary neuroendocrine tumors, we examined the expression of $d r s$ mRNA in tissue specimens from 3 cases of TC, 4 cases of AC, 2 cases of LCNEC, and 11 cases of SCLC by in situ mRNA hybridization. Four cases of normal lung and bronchial epithelia, 8 samples of normal brain tissue, and 2 cases of tumorlets in the lung were also examined. The drs mRNA was definitely expressed in all normal tissues of the lung and brain, and $3 \mathrm{TC}$ and 2 tumorlet tissues. The expression of $d r s$ mRNA
\end{abstract}

Correspondence to: Dr Misuzu Shimakage, Department of Pediatrics, National Hospital Organization, Wakayama National Hospital, Mihama-cho, Hidaka-gun, Wakayama 644-0044, Japan E-mail: misuzu_s@wakayama2.hosp.go.jp

Abbreviations: TC, typical carcinoid; AC, atypical carcinoid; LCNEC, large-cell neuroendocrine carcinoma; SCLC, small-cell lung carcinoma

Key words: drs, neuroendocrine tumor, small-cell lung carcinoma, pulmonary carcinoid, in situ hybridization was also detected in 2 of 2 LCNEC tissues and 3 of 4 AC tissues, although the signals were weak. On the other hand, drs mRNA was not detected in 10 of 11 SCLC tissues. Downregulation of $d r s$ mRNA was also observed in 3 of 4 SCLC cell lines that were examined by reverse transcriptasepolymerase chain reaction (RT-PCR). Neither gross deletion nor rearrangement of the $d r s$ genome was detected in these cell lines by Southern blot analysis. Our results indicate that the downregulation of $d r s$ is correlated with the development of SCLC, a highly malignant pulmonary neuroendocrine tumor.

\section{Introduction}

Small-cell lung carcinoma (SCLC) has been reported to show neuronal characteristics including many neuronal markers, neuronal cell adhesion molecules, a neuron-restrictive silencer factor, neurotransmitters, and voltage-gated ion channels, namely, membrane excitability (1). Lung neuroendocrine tumors include the following tumor types: typical carcinoid tumor (TC), atypical carcinoid tumor (AC), large cell neuroendocrine carcinoma (LCNEC), and small-cell lung carcinoma $(2,3)$. The five-year survival rate of patients with these tumors was reported to be $87-100 \%$ in TC, $37-71 \%$ in AC, $15-45 \%$ in LCNEC, and $0-20 \%$ in SCLC (3). Although the five-year survival rate decreases from TC to SCLC, each tumor type is considered to develop independently and not to progress from one type to another. Almost all carcinoid tumors in the lung show few symptoms, and they are rarely resected. Studies of the genetic alterations of proto-oncogenes and tumor suppressor genes, including myc, PTEN, $p 53$, and $R b$, in SCLC have been performed (4-6). Various genetic changes such as chromosomal loss, mutations, and methylation were also reported for other carcinoid tumors, including TC, $\mathrm{AC}$, and LCNEC (7-12). However, analyses of the expression of oncogenes or tumor suppressor genes in pulmonary 
neuroendocrine tumors covering all four categories have been scarcely reported.

The $d r s$ gene was originally isolated as a suppressor of $\mathrm{v}$-src transformation $(13,14)$. The expression of $d r s$ mRNA has been shown to be markedly downregulated in a variety of human cancer cell lines (15) and malignant tumor tissues, including colon cancer $(16,17)$, pulmonary adenocarcinoma (18), prostatic carcinoma (19), and adult T-cell leukemia/ lymphoma (20), suggesting the potential role of this gene as a tumor suppressor. We also found that Drs protein interacted with ASY/Nogo-B, an apoptosis-inducing protein localized in the endoplasmic reticulum, and that ectopic expression of the Drs protein induced apoptosis in human cancer cells without mitochondrial involvement (21). Furthermore, malignant tumors including T-cell lymphomas, lung adenocarcinomas and hepatomas were generated in $\sim 30 \%$ of the $d r s$ knockout mice (22). All these findings indicate that $d r s$ is involved in the suppression of malignant tumor formation by promoting apoptosis.

In this study, to clarify the role of the $d r s$ gene in the development of neuroendocrine tumors in the lung, we investigated the expression level of $d r s$ mRNA in tissue specimens from four categories of human pulmonary neuroendocrine tumors by in situ hybridization and in SCLC cell lines by the reverse transcriptase-polymerase chain reaction (RT-PCR). It is necessary to examine series of all these pulmonary neuroendocrine tumors employing a standardized technique. Our results showed that the downregulation of $d r s$ is correlated with the malignancy of pulmonary neuroendocrine tumors, and suggested that $d r s$ played a role in the development of SCLC.

\section{Patients and methods}

Patients. Twenty patients with neuroendocrine tumors in the lung were selected from the neuroendocrine files (19952007) of Osaka Prefectural Medical Center for Cancer and Cardiovascular Diseases, and Osaka Prefectural Medical Center for Respiratory and Allergic Diseases. No cases exhibited features indicative of an immunocompromised state. The mean age of the neuroendocrine tumor patients was 64.3 years. The clinical classification was in accordance with the general rules indicated in previous studies $(2,3)$.

Cell lines. The human SCLC cell lines used in this study were H69, Lu139, LC22, and REFE-LC-MS. Normal human fibroblasts (HF) were also used as a positive control for the expression of $d r s$ mRNA. These cells were cultured in RPMI1640 medium supplemented with $10 \%$ fetal calf serum.

In situ mRNA hybridization. Formalin-fixed paraffin-embedded tissues were cut in serial sections of a $3-\mathrm{mm}$ thickness. As a probe, human $d r s$ cDNA was cloned into the plasmid, pBluescript II SK(-). The sense and antisense RNA probes were labeled with digoxigenin-11-UTP by in vitro transcription using a commercial kit (Roche-Boehringer-Mannheim, Indianapolis, IN, USA). The labeled riboprobes were then fragmented to $\sim 100$ bases in length by alkaline hydrolysis. The sense probe served as a negative control. In situ mRNA hybridization was performed as described previously
$(16,18-20)$. The adjacent sections of the paraffin blocks were stained with hematoxylin and eosin (H\&E).

$R T-P C R$. Reverse transcription was carried out using total RNA $(2 \mu \mathrm{g})$ from the cell lines. Oligo(dT)-adaptor primers (Invitrogen) were employed for the reverse transcription reaction. The reverse transcription products were amplified through 30 cycles using degenerate primers $(0.4 \mu \mathrm{M})$, and Takara Ex Taq polymerase (2.5 units). The primer sets employed for PCR to detect human drs mRNA were 5'-TTA AGTGAGCTGTGCAGCCT-3' for the forward primer (pDrsU2) and 5'-TAACAGCACATCAGACGTTGC-3' for the reverse primer ( $\mathrm{pDrsSR} 3$, which is located in the $3^{\prime}$ downstream of the consensus repeat 2 of $d r s$ ). The primer sets used to detect glyceraldehyde-3-phosphodehydrogenase (GAPDH) were 5'-CTGCAAGGGGCTGATAAGAG-3' for the forward primer and 5'-AGGGGTCAGCCTTGTTTTCT-3' for the reverse primer. Amplicons were separated in $1.5 \%$ agarose gel.

Southern blot analysis. Genomic DNA from the cell lines was digested with restriction enzymes, separated by $1 \%$ agarose gel electrophoresis, and transferred to a nylon filter. The filter was then hybridized with a ${ }^{32} \mathrm{P}$-labeled probe at $42^{\circ} \mathrm{C}$ overnight in hybridizing buffer $(50 \%$ formamide, $0.6 \mathrm{M}$ sodium chloride, $60 \mathrm{mM}$ sodium citrate, $0.2 \%$ SDS, $0.1 \%$ bovine serum albumin, $0.1 \%$ Ficoll, $0.1 \%$ polyvinylpyrrolidone, and $50 \mu \mathrm{g}$ of herring sperm DNA/ml). The hybridized filter was washed with $15 \mathrm{mM}$ sodium chloride, $1.5 \mathrm{mM}$ sodium citrate, and $0.1 \% \mathrm{SDS}$ at $55^{\circ} \mathrm{C}$, and then autoradiographed.

\section{Results}

To clarify the correlation between the downregulation of $d r s$ mRNA and development of neuroendocrine tumors in the lung, we investigated the expression of $d r s$ mRNA in tissues of four categories of human neuroendocrine tumors, including TC, AC, LCNEC, and SCLC, by in situ mRNA hybridization. Tissue specimens of 3 cases of TC, 4 cases of AC, 2 cases of LCNEC, and 11 cases of SCLC were prepared from 20 patients. For a comparative study, normal lung, bronchial and neuronal tissues, and pulmonary tumorlets, which are suspected to be noncancerous growths of Kultschitzky cells, were also prepared.

The results of $d r s$ mRNA in situ hybridization are summarized in Table I, and representative pictures of the in situ hybridization with sense and antisense probes and H\&E staining are shown in Fig. 1. drs mRNA was definitively expressed in the cytoplasm of normal human brain, lung, and bronchus tissues with antisense probes (Fig. 1a, c, and e, respectively), but was not detected with sense probes (Fig. 1b and d). The large pyramidal cells (Fig. 1a) and type I pneumocytes (Fig. 1c) showed distinct signals. The bronchial epithelial cells also exhibited strong signals (Fig. 1e). In the tissues examined from 2 tumorlets, clear $d r s$-positive signals were detected with antisense probes (Fig. 1f). In 3 cases of typical carcinoid (TC) tissues, strong signals were detected (Fig. 1g).

In 4 cases of atypical carcinoid (AC), one case (case 1) showed strong signals and 2 cases (cases 2 and 3 ) showed 


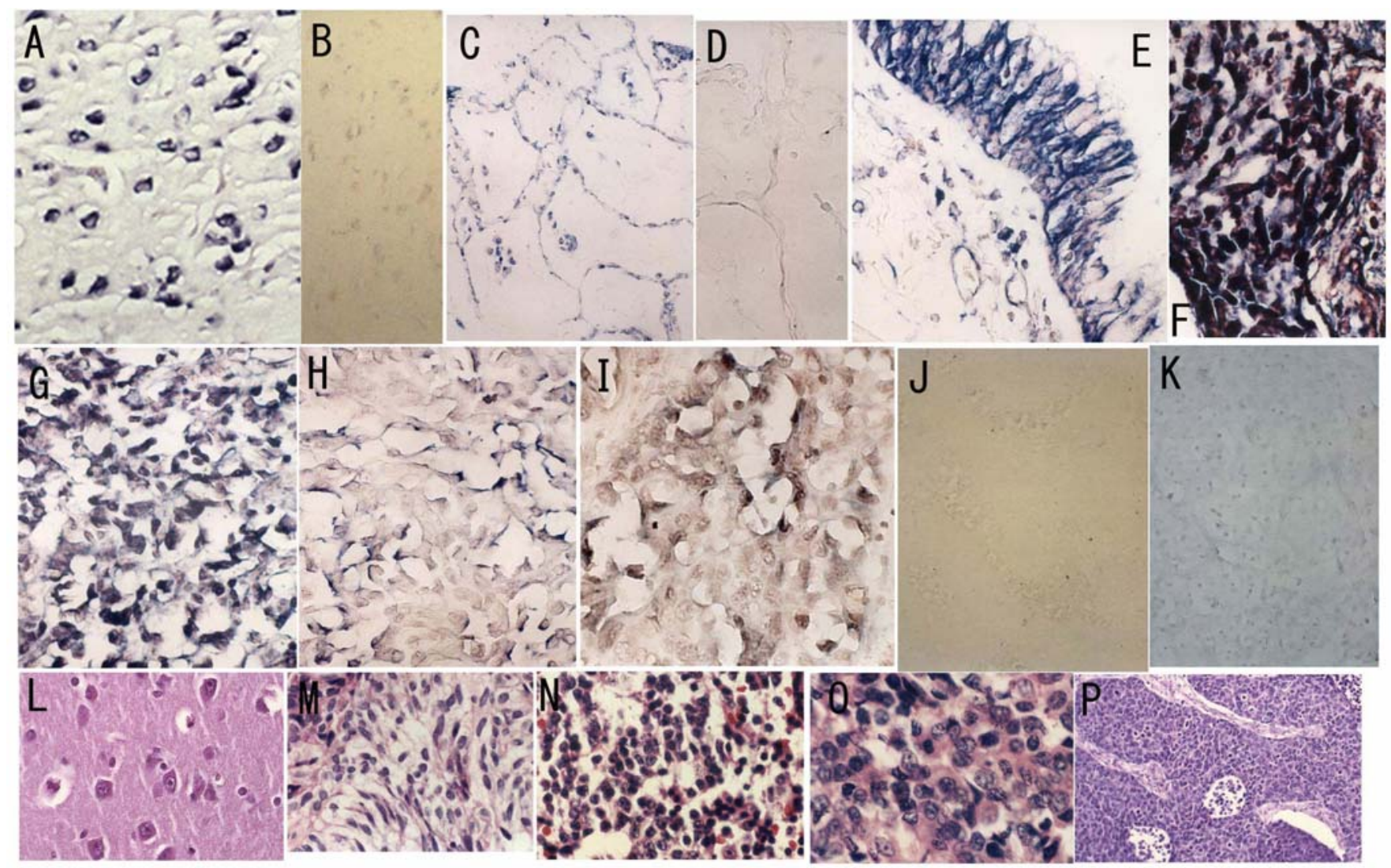

Figure 1. In situ hybridization analyses of the expression of drs mRNA in human pulmonary neuroendocrine tumors. (a-k) In situ hybridization; a, hippocampus with antisense probe, x200; b, hippocampus with sense probe, x200; c, normal lung (case 1) with antisense probe, x200; d, normal lung (case 1) with sense probe, x200; e, normal bronchus with antisense probe, x200; f, tumorlet (case 2) with antisense probe, x200; g, typical carcinoid tumor (case 1) with antisense probe, $\mathrm{x} 200$; h, atypical carcinoid tumor (case 1) with antisense probe, x200; i, LCNEC (case 2) with antisense probe, x200; j, SCLC case (case 4) with antisense probe, $\mathrm{x} 200 ; \mathrm{k}$, SCLC (case 11) with antisense probe, $\mathrm{x} 200$; (1-p) hematoxylin and eosin (H\&E) staining; 1, hippocampus, x200; m, tumorlet (case 2), x200; n, typical carcinoid tumor (case 1), x200; o, LCNEC (case 2), x200; p, SCLC (case 4), x200.

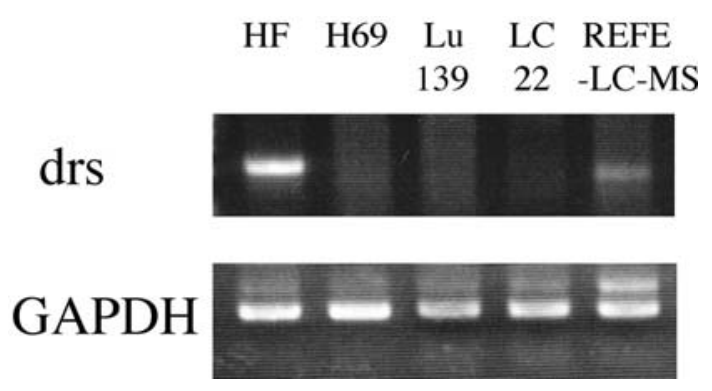

Figure 2. RT-PCR analysis of the expression of $d r s$ mRNA in SCLC cell lines. RT-PCR was performed as described in Patients and methods. The expression of glyceraldehyde-3-phosphodehydrogenase (GAPDH) was used as an internal control.

weak signals (Fig. 1h), but the other case (case 4) exhibited no signals (see Table I). In the two large cell neuroendocrine tumors (LCNEC) examined, $d r s$-positive signals were detected, although one case showed weak signals (Fig. 1i). In neuroendocrine tumors, including TC, AC, and LCNEC, which showed a relatively high five-year survival rate, $d r s$ mRNA was mostly expressed. On the other hand, the expression of $d r s$ mRNA was not detected in almost any of the (10/11) small-cell lung carcinoma (SCLC) tissues examined (Fig. 1j and k). Only one case (case 1) of SCLC exhibited very weak signals.

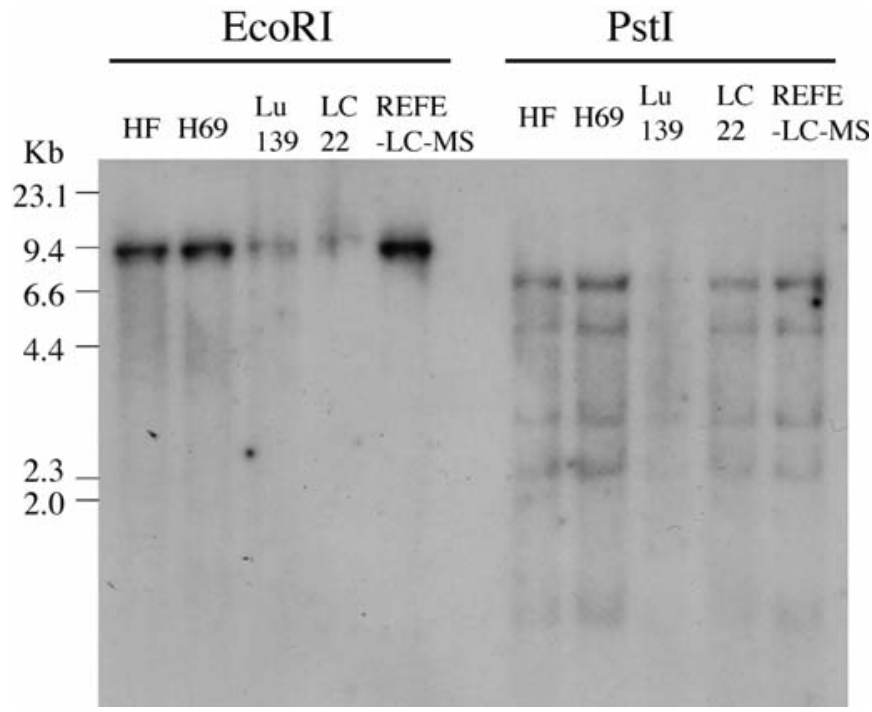

Figure 3. Southern blot hybridization. EcoRI- or PstI-digested genomic DNA, prepared from SCLC cell lines (H69, Lu139, LC22, and REFE-LCMS) and normal human fibroblasts (HF), was hybridized with a human $d r s$ cDNA probe.

To confirm the results of $d r s$ mRNA expression in SCLC tissues, we also investigated the expression of $d r s$ mRNA in four SCLC cell lines (H69, Lu139, LC22, and REFE-LC-MS) 
Table I. In situ mRNA hybridization.

\begin{tabular}{|c|c|c|c|c|c|c|}
\hline Disease & Case & Age & Gender & TMN & Stage & drs \\
\hline \multirow[t]{8}{*}{ Normal brain } & Mid-brain & 54 & $\mathrm{~F}$ & & & ++ \\
\hline & Pons & 54 & $\mathrm{~F}$ & & & ++ \\
\hline & Medulla & 54 & $\mathrm{~F}$ & & & ++ \\
\hline & Temporal lobe & 54 & $\mathrm{~F}$ & & & ++ \\
\hline & Frontal lobe & 54 & $\mathrm{~F}$ & & & ++ \\
\hline & Basal ganglia & 54 & $\mathrm{~F}$ & & & ++ \\
\hline & Dentate nucleus & 54 & $\mathrm{~F}$ & & & ++ \\
\hline & Hippocampus & 54 & $\mathrm{~F}$ & & & ++ \\
\hline Normal bronchus & 1 & 51 & M & & & ++ \\
\hline \multirow[t]{3}{*}{ Normal lung } & 1 & 51 & M & & & ++ \\
\hline & 2 & 70 & $\mathrm{~F}$ & & & ++ \\
\hline & 3 & 44 & $\mathrm{~F}$ & & & ++ \\
\hline \multirow[t]{2}{*}{ Tumorlet } & 1 & 68 & M & & & ++ \\
\hline & 2 & 53 & M & & & + \\
\hline \multirow[t]{7}{*}{ Carcinoid tumor } & Typical & 62 & M & T1N0M0 & $1 \mathrm{~A}$ & ++ \\
\hline & Typical & 70 & M & T2N0M0 & 1B & ++ \\
\hline & Typical & 69 & M & T1N0M0 & $1 \mathrm{~A}$ & + \\
\hline & Atypical & 70 & M & T1N0M0 & $1 \mathrm{~A}$ & + \\
\hline & Atypical 2 & 77 & M & $\mathrm{T} 2 \mathrm{~N} 2 \mathrm{M} 0$ & $3 \mathrm{~A}$ & Weakly + \\
\hline & Atypical 3 & 49 & M & T2NOM0 & $1 \mathrm{~A}$ & Weakly + \\
\hline & Atypical 4 & 64 & $\mathrm{~F}$ & T1N0M0 & $1 \mathrm{~B}$ & - \\
\hline \multirow[t]{2}{*}{ LCNEC } & 1 & 64 & M & T2N1M0 & $1 \mathrm{~B}$ & + \\
\hline & 2 & 62 & M & T2NOM0 & $1 \mathrm{~B}$ & Weakly + \\
\hline \multirow[t]{11}{*}{ SCLC } & 1 & 58 & M & T1N0M0 & $1 \mathrm{~A}$ & Weakly + \\
\hline & 2 & 51 & M & $\mathrm{T} 1 \mathrm{~N} 2 \mathrm{M} 0$ & $3 \mathrm{~A}$ & - \\
\hline & 3 & 51 & M & T2N0M0 & $1 \mathrm{~B}$ & - \\
\hline & 4 & 72 & M & $\mathrm{T} 1 \mathrm{~N} 2 \mathrm{M} 0$ & $3 \mathrm{~A}$ & - \\
\hline & 5 & 71 & M & T1N0M0 & $1 \mathrm{~A}$ & - \\
\hline & 6 & 75 & M & T3N3M0 & $3 \mathrm{~B}$ & - \\
\hline & 7 & & & & & - \\
\hline & 8 & & & & & - \\
\hline & 9 & & & & & - \\
\hline & 10 & & & & & - \\
\hline & 11 & & & & & - \\
\hline
\end{tabular}

by RT-PCR. Human fibroblasts (HF) were also used as a positive control. As shown in Fig. 2, the expression of $d r s$ mRNA was not detected in 3 of the 4 SCLC cell lines examined (H69, Lu139, and LC22). Only one cell line, REFELC-MS, showed a weak signal compared with the strong signal seen in HF cells. This RT-PCR result was also confirmed by the in situ mRNA hybridization method (data not shown).

To examine the status of the $d r s$ genome in these SCLC cell lines, Southern blot hybridization was performed using the same cell lines. As shown in Fig. 3, no large deletions or rearrangements were detected in the genome of the $d r s$ gene in SCLC cell lines whose levels of $d r s$ mRNA were markedly reduced (H69, Lu139, and LC22). Our results indicate that the expression of $d r s$ mRNA was markedly downregulated in most malignant SCLC tissues and cell lines, suggesting that the downregulation of $d r s$ plays a role in the development of SCLC.

\section{Discussion}

In the present study, we investigated the expression of the $d r s$ tumor suppressor gene in four categories of human pulmonary neuroendocrine tumors, and found that the expression of $d r s$ mRNA was markedly downregulated in most SCLC tissues and cell lines among these neuroendocrine tumors. As for other 
categories of neuroendocrine tumors, including TC, AC, and LCNEC, drs mRNA was clearly expressed in most of these tumor tissues, although the expression was weak in some of them, especially in AC and LCNEC tumors.

Considering the malignancy of these four categories of neuroendocrine tumors, as estimated by the five-year survival rate of patients (3), these results suggest that the downregulation of the $d r s$ gene is correlated with the malignancy of neuroendocrine tumors, because drs downregulation was most evident in malignant SCLC tumors (five-year survival rate: $0-20 \%$ ), and $d r s$ mRNA was definitely expressed in all cases of TC tumors (five-year survival: $87-100 \%$ ) examined. Since TC is less malignant and frequently classified as a group containing tumorlets (23), we examined the sum of these two categories. As a result, a significant difference was seen in the ratio of cases with $d r s$ mRNA expression between SCLC $(1 / 11)$ and tumorlet plus TC $(5 / 5)\left(\mathrm{p}=0.0005\right.$ by $\chi^{2}$ examination; $\mathrm{p}=0.0014$ by Fisher's examination).

In $\mathrm{AC}$ and LCNEC, which show moderate five-year survival rates $(37-71 \%$ and $15-45 \%$, respectively), the expression of $d r s$ mRNA was relatively weak. As for AC tumors, the level of atypia varied across tumors. This result may reflect the expression level of $d r s$ mRNA. The expression of $d r s$ mRNA was not correlated with the clinical stage or TNM classification, at least in the neuroendocrine tumors examined. These results are similar to those in our previous in situ hybridization experiments on colon and lung adenocarcinomas $(16,18)$. drs mRNA was strongly expressed in colon adenoma with mild atypia, but was downregulated in adenomas with moderate atypia and adenocarcinoma. The downregulation of $d r s$ mRNA was also correlated with a poor degree of differentiation and the progression of prostatic carcinoma (19). The present study showed that the link between $d r s$ downregulation and malignancy is also applicable to neuroendocrine tumors, although the latter do not progress.

Originally, the $d r s$ gene was found to be downregulated in a rat fibroblast cell line by retroviral oncogenes such as $\mathrm{v}$-src, v-ras, v-sis, and v-mos (13). Therefore, a possible explanation for the present findings is that the expression of the drs gene is downregulated by the activation of endogenous oncogenes in SCLC. In fact, it has been reported that myc is overexpressed by gene amplification in SCLC (5). Loss of heterozygosity (LOH) of PTEN, which activates Akt, was also frequently observed in SCLC cell lines (4). Recently, it was reported that Pyk2 and Src kinase activation plays an essential role in the neuropeptide-induced proliferation of SCLC cells (24).

Our study revealed differential $d r s$ expression in neuroendocrine tumors in the lung, and the loss of drs function in SCLC, suggesting that $d r s$ is involved on the development of SCLC. Previously, it has been reported that SCLC derived from bronchial epithelial cells, whereas, typical carcinoid derived from neural crest cells (25). Therefore, SCLC is suspected to be different from other neuroendocrine tumors. On the other hand, the evidence indicating that low grade neuroendocrine carcinoma (atypical carcinoid) shows intermediate characterictics between SCLC and typical carcinoid was reported (26). Our results presented herein agreed with these studies. Further investigation, including the detection of small mutations and methylation of the drs genome in SCLC tissues and cell lines, is necessary to clarify the mechanism of the downregulation of $d r s$ mRNA in the genesis of SCLC.

\section{Acknowledgements}

This study was supported by a Grant-in-Aid for Cancer Research (15-4) from the Ministry of Health, Labour and Welfare of Japan (to M. Shimakage). This project was also supported by Grants-in-Aid for Scientific Research (C) (Grant No. 17590341 and No. 19590388) from the Ministry of Education, Science, Sports, and Culture of Japan (to H. Inoue).

\section{References}

1. Onganer PU, Seckl MJ and Djamgoz MBA: Neuronal characteristics of small-cell lung cancer. Br J Cancer 93: 1197-1201, 2005.

2. Onuki N, Wistuba II, Travis WD, et al: Genetic changes in the spectrum of neuroendocrine lung tumors. Cancer 85: 600-607, 1999.

3. Hage R, Brutel de la Riviere A, Seldenrijk CA, et al: Update in pulmonary carcinoid tumors: A review article. Ann Surg Oncol 10: 697-704, 2003.

4. Jackman DM and Johnson BE: Small-cell lung cancer. Lancet 366: 1385-1396, 2005

5. Pisick E, Jagadeesh S and Salgia R: Small cell lung cancer: from molecular biology to novel therapeutics. J Exp Ther Oncol 3: 305-318, 2003.

6. Cagle PT, El-Naggar AK and Xu H-J: Differential retinoblastoma protein expression in neuroendocrine tumors of the lung. Am J Pathol 150: 393-400, 1997.

7. Leotlela PD, Jauch A, Holtgreve-Grez H, et al: Genetics of neuroendocrine and carcinoid tumors. Endocr Relat Cancer 10: 437-450, 2003

8. Sachithanandan N, Harle RA and Burgess JR: Bronchopulmonary carcinoid in multiple endocrine neoplasia type 1. Cancer 103: 509-515, 2004

9. Finkelstein SD, Hasegawa T, Colby T, et al: 11q13 allelic imbalance discriminates pulmonary carcinoids from tumorlets. Am J Pathol 155: 633-640, 1999.

10. Walch AK, Zitzelsberger HF, Aubele MM, et al: Typical and atypical carcinoid tumors of the lung are characterized by $11 \mathrm{q}$ deletions as detected by comparative genomic hybridization. Am J Pathol 153: 1089-1098, 1998.

11. Haruki N, Yatabe Y, Travis WD, et al: Characterization of highgrade neuroendocrine tumors of the lung in relation to menin mutations. Jpn J Cancer Res 91: 317-323, 2000.

12. Shin J H, Kang SM, Kim YS, et al: Identification of tumor suppressor loci on the long arm of chromosome 5 in pulmonary large cell neuroendocrine carcinoma. Chest 128: 2999-3003, 2005.

13. Pan J, Nakanishi K, Yutsudo M, et al: Isolation of a novel gene down-regulated by v-src. FEBS Lett 383: 21-25, 1996.

14. Inoue H, Pan J and Hakura A: Suppression of v-src transformation by the $d r s$ gene. J Virol 72: 2532-2537, 1998.

15. Yamashita A, Hakura A and Inoue H: Suppression of anchorageindependent growth of human cancer cell lines by the $d r s$ gene. Oncogene 18: 4777-4778, 1999.

16. Shimakage M, Kawahara K, Kikkawa N, et al: Down-regulation of drs mRNA in human colon adenocarcinomas. Int J Cancer 87: 5-11, 2000.

17. Mukaisho K, Suo M, Shimakage M, et al: Down-regulation of drs mRNA in colorectal neoplasms. Jpn J Cancer Res 93: 888-893, 2002

18. Shimakage M, Takami K, Kodama K, et al: Expression of drs mRNA in human lung adenocarcinoma. Hum Pathol 33: 615-619, 2002.

19. Kim CJ, Shimakage M, Kushima R, et al: Down-regulation of drs mRNA in human prostate carcinomas. Hum Pathol 34: 654-657, 2003

20. Shimakage M, Inoue N, Ohshima K, et al: Downregulation of $d r s$ mRNA expression is associated with the progression of adult T-cell leukemia/lymphoma. Int J Oncol 30: 1343-1348, 2007. 
21. Tambe $\mathrm{Y}$, Isono $\mathrm{T}$, Haraguchi $\mathrm{S}$, et al: A novel apoptotic pathway induced by the drs tumor suppressor gene. Oncogene 23: 2977-2987, 2004.

22. Tambe Y, Yoshioka-Yamashita A, Mukaisho K, et al: Tumor prone phenotype of mice deficient in a novel apoptosis-inducing gene, drs. Carcinogenesis 28: 777-784, 2007.

23. Torikata C: Tumorlet of the lung - an ultrastructural study. Ultrastruct Pathol 15: 189-195, 1991.

24. Roelle S, Grosse R, Buech T, et al: Essential role of Pyk2 and Src kinase activation in neuropeptide-induced proliferation of small cell lung cancer cells. Oncogene 27: 1737-1748, 2008.
25. Anbazhagan R, Tihan T, Bornman DM, et al: Classification of small cell lung cancer and pulmonary carcinoid by gene expression profiles. Cancer Res 59: 5119-5122, 1999.

26. Helpap B and Kollermann J: Immunohistochemical analysis of the proliferative activity of neuroendocrine tumors from various organs. Are there indications for a neuroendocrine tumorcarcinoma sequence? Virchows Arch 438: 86-91, 2001. 